\title{
Práticas educativas e a formação para Atenção Primária: O Médico como educador
}

\section{em Saúde}

\author{
Educational practices and training for Primary Care: The Physician as Health educator \\ Prácticas educativas y formación para la Atención Primaria: El Médico como educador Sanitario
}

Recebido: 21/05/2021 | Revisado: 29/05/2021 | Aceito: 01/06/2021 | Publicado: 14/06/2021

Vivianne de Lima Biana Assis
ORCID: https://orcid.org/0000-0002-5110-7698
Universidade Federal de Sergipe, Brasil
E-mail: vivibiana@gmail.com
Universidade Estadual de Ciências da Saúde de Alagoas, Brasil
Marinília Cristina Barbosa Fernandes
ORCID: https://orcid.org/0000-0002-0271-8791
Centro Universitário Tiradentes, Brasil
E-mail: mariniliab@gmail.com
Jaqueline Teixeira Silva Valença
ORCID: https://orcid.org/0000-0002-1103-8229
Centro Universitário Tiradentes, Brasil
E-mail: jaquelineteixeira95@gmail.com
Divaldo Pereira Lyra Junior
ORCID: https://orcid.org/0000-0002-0266-0702
Universidade Federal de Sergipe, Brasil
E-mail: lyra_jr@ @hotmail.com

\begin{abstract}
Resumo
Objetivo: analisar as práticas educativas realizadas na Atenção Primária à Saúde pelos estudantes de Medicina das universidades públicas e privadas do município de Maceió, em nível de planejamento e execução. Métodos: Trata-se de um estudo empírico de natureza descritiva e exploratória, de abordagem qualitativa. Foram entrevistados discentes do curso de Medicina, de universidades públicas e privadas, inseridos nos cenários de prática da Atenção Primária no ano de 2019 e 2020. Utilizou-se o método da Entrevista Aberta com questões norteadoras e para analisar o conteúdo da mensagem, foi considerado cada oração como Unidades de Registro. Resultados: Foram entrevistados 38 estudantes com média de idade $24,81 \pm 4,91$ sendo $60,5 \%$ do sexo feminino, do total $21 \%$ pertencem ao décimo período do curso, 23,7\% possuíam formação anterior completa ou incompleta, sendo 73,7\% estudantes de Instituição privada. Foi possível identificar as seguintes categorias "Ações Educativas Desenvolvidas", "Recursos Educativos"; "Integração, Ensino, Serviço e Comunidade"; "Trabalho em Equipe"; "Prevenção a Saúde"; "Autocuidado"; "Benefícios das ações Educativas"; "Dificuldades das ações Educativas"; "Sugestões das ações Educativas". Com base na análise das categorias evidenciou-se três eixos temáticos: "Vivência Prática", "Cuidado em Saúde" e "Ações Educativas". Considerações Finais: Através da análise do discurso, este estudo compreendeu a percepção do estudante de medicina acerca das práticas educativas oportunizadas na Atenção Primária evidenciando que a educação em saúde proporcionou aproximação da realidade comunitária, criação do vínculo com os usuários e equipe de saúde, valorização do trabalho em equipe e reconhecimento do papel do médico neste cenário.
\end{abstract}

Palavras-chave: Atenção primária a saúde; Educação em saúde; Ensino superior.

\begin{abstract}
Objective: To analyze the educational practices carried out in Primary Health Care by medical students from public and private universities in the municipality of Maceió, in terms of planning and execution. Methods: This is an empirical study of a descriptive and exploratory nature, with a qualitative approach. Medical school students from public and private universities, inserted in Primary Care practice settings in 2019 and 2020, were interviewed. The Open Interview method was used with guiding questions and to analyze the content of the message, each sentence was considered as Units of Record. Results: 38 students were interviewed with mean age $24.81 \pm 4.91$ being $60.5 \%$ female, of the total $21 \%$ belonged to the tenth period of the course, $23.7 \%$ had complete or incomplete previous education, being $73.7 \%$ students from a private institution. It was possible to identify the following categories "Developed Educational Actions", "Educational Resources"; "Integration, Teaching, Service and Community"; "Teamwork"; "Health Prevention"; "Self-Care"; "Benefits of Educational Actions"; "Difficulties of Educational Actions"; "Suggestions for Educational Actions". Based on the analysis of the categories, three thematic axes were highlighted: "Practical Experience", "Health Care" and "Educational Actions". Final Considerations: Through discourse analysis, this study understood the medical student's perception about the educational practices offered in
\end{abstract}


Primary Care, showing that health education provided approximation to the community's reality, creation of a bond with users and health team, appreciation of team work and recognition of the physician's role in this scenario.

Keywords: Primary health care; Health education; Higher education.

\section{Resumen}

Objetivo: analizar las prácticas educativas realizadas en la Atención Primaria por los estudiantes de Medicina de las universidades públicas y privadas del municipio de Maceió, en el nivel de planificación y ejecución. Métodos: un estudio empírico con un enfoque cualitativo. Fueron entrevistados discentes del curso de Medicina, de universidades públicas y privadas, insertados en los cenários de prática de la Atención Primaria en el año de 2019 y 2020 . Se utilizó el método de la Entrevista Aberta con preguntas norteadoras. Resultados: Se entrevistaron 38 alumnos con una edad media de 24,81 $\pm 4,91$ siendo el $60,5 \%$ mujeres, del total el $21 \%$ pertenecía al décimo periodo del curso, el $23,7 \%$ tenía estudios previos completos o incompletos, siendo el 73,7\% alumnos de Institución privada. Fue posible identificar las siguientes categorías "Acciones educativas desarrolladas", "Recursos educativos"; "Integración, enseñanza, servicio y comunidad"; "Trabajo en equipo"; "Prevención de la salud"; "Autocuidado"; "Beneficios de las acciones educativas"; "Dificultades de las acciones educativas"; "Sugerencias de acciones educativas". A partir del análisis de las categorías, se destacaron tres ejes temáticos: "Experiencia práctica", "Atención sanitaria" y "Acciones educativas". Consideraciones finales: A través del análisis del discurso, este estudio comprendió la percepción de los estudiantes de medicina sobre las prácticas educativas impartidas en la Atención Primaria, mostrando que la educación en salud proporcionó una aproximación a la realidad de la comunidad, la creación de un vínculo con los usuarios y el equipo de salud, la valoración del trabajo en equipo y el reconocimiento del papel del médico en este escenario.

Palabras clave: Atención primaria de salud; Educación en salud; Educación superior.

\section{Introdução}

As alterações do perfil demográfico e epidemiológico da população exigiu mudanças no sistema de saúde e no perfil dos profissionais de saúde. A criação da Atenção Primária à Saúde (APS) se fundamenta na integralidade do cuidado e a promoção se relaciona com uma de suas responsabilidades, por caracterizar-se como forma prática e conceitual de políticas públicas afim de possibilitar autonomia e incentivar o autocuidado indivíduo e coletivo. Logo, a aproximação da saúde com a educação pode repensar e reorientar as práticas profissionais, a organização dos serviços e a gestão do sistema, apontando para a construção de políticas (Barreto et al., 2019; Nutbeam, 2019; Ribeiro, 2019).

No Brasil, a transição demográfica trouxe demandas específicas, exigindo respostas oportunas (Miranda et al., 2017). A aprovação da Política Nacional da Atenção Básica centra a gestão e a coordenação da Rede de Atenção à Saúde nas pessoas, de forma acolhedora, resolutiva e que utiliza tecnologias complexas que auxiliam o manejo de necessidades de saúde mais prevalentes e relevantes, observando critérios de risco, vulnerabilidade, resiliência e ética (Brasil, 2017). Logo, o Sistema Único de Saúde (SUS) requer profissionais empenhados com princípios de equidade e cuidado integral, com formação direcionada para prática de atenção à saúde, com ênfase na APS (Vendruscolo et al., 2016).

Ao longo dos anos, o ensino em saúde tem enfrentado múltiplos desafios para adaptar a formação dos profissionais de saúde para as necessidades atuais. Nesse cenário, a universidade ocupa o espaço legítimo da produção científica e de subjetivação, em que escolas médicas compõem instituições complexas que articulam múltiplos sujeitos, processos e disputas que influenciam na produção do saber (Feuerwerker, 2014). Assim, as práticas da educação em saúde na APS compõem o processo de trabalho das equipes, ampliando as bases conceituais destas práticas e os processos de subjetivação que se movimentam no território.

Diante disso, as práticas educativas têm apresentado mudanças nas ferramentas para comunicação transformada pela comunicação digital complexa. Essas mudanças devem refletir o conteúdo e o modo de entrega da educação em saúde para se envolver de forma significativa com determinantes sociais e econômicos da saúde (Soares et al., 2017; Nutbeam, 2019). A partir da mudança do ensino em saúde e da valorização APS como ordenadora do cuidado, este estudo tem como objetivo de analisar práticas educativas realizadas na APS pelos estudantes de Medicina em uma cidade do Nordeste do Brasil. 


\section{Metodologia}

Trata-se de um estudo empírico de natureza descritiva e exploratória, de abordagem qualitativa, que permite a compreensão de valores culturais e representações de determinado grupo acerca de um tema específico, sob diferentes perspectivas, abordando as relações entre atores sociais e ações implementadas (Bosi, 2004; Minayo, 2011). Participaram deste estudo discentes do curso de Medicina, de universidades públicas e privadas, do município de Maceió - Alagoas, inseridos nos cenários de prática da APS no ano de 2019 e 2020.

Os sujeitos sociais foram considerados em número suficiente, de tal forma que permitiram a reincidência das informações, sem desprezar informações relevantes. O conjunto de informantes diversificado possibilitou a apreensão de semelhanças e diferenças (Minayo, 2011). Foram excluídos desta pesquisa estudantes que realizaram apenas visita técnica ou que realizavam apenas atividades observacionais nas unidades de saúde. Para permitir que o entrevistador tenha liberdade para desenvolver situações e explorar amplamente a questão desejada, optou-se pelo método da Entrevista Aberta ou em Profundidade com questões norteadoras.

As entrevistas ocorreram em local previamente acordado entre as partes e devido a pandemia Covid-19 algumas entrevistas foram realizadas via plataforma virtual google meeting. Para caracterização dos sujeitos estudados, foi solicitado o preenchimento de um questionário, contendo informações de identificação pessoal, período cursado, disciplina vinculada a atividade prática, formação escolar e/ou profissional onde o próprio entrevistado pode preencher.

Este estudo utilizou a análise de conteúdo de Bardin (1977) que, dentre os diversos tipos de análise de conteúdo, a modalidade de análise temática se apresenta como uma ferramenta que associa o tema a uma afirmação a respeito de determinado assunto. Este, por sua vez, comporta um feixe de relações e pode ser graficamente apresentado por meio de uma palavra, uma frase, um resumo.

O conteúdo das mensagens foi obtido por meio da decomposição do conjunto do texto (cada oração ou frase) em unidades de registro (UR). Desta forma, cada UR recebeu uma codificação alfanumérica, na qual o código alfabético identifica o sujeito analisado, enquanto o código numérico identifica a UR da fala deste sujeito. Para a interpretação dos dados levantados, inicialmente as unidades foram agrupadas em categorias e, no segundo momento, as categorias foram comparadas entre si, para assim serem agrupadas em eixos temáticos.

A pesquisa foi aprovada pelo parecer $n^{\text {o } 3.539 .429 ~ d o ~ C o m i t e ̂ ~ d e ~ E ́ t i c a ~ e m ~ P e s q u i s a ~ d o ~ C e n t r o ~ U n i v e r s i t a ́ r i o ~}$ Tiradentes - UNIT-AL. Durante o estudo, os sujeitos da pesquisa assinaram um termo de consentimento livre e esclarecido, que lhes assegurou anonimato, confidencialidade dos dados pessoais e a utilização das informações somente para fins científicos.

\section{Resultados e Discussão}

Foram entrevistados 38 estudantes, sendo esses adultos jovens, com média de idade 24,81 $\pm 4,91$, predominantemente solteiros $(94,7 \%)$ e $60,5 \%$ do sexo feminino. Do total, $21 \%$ pertencem ao décimo período do curso, 23,7\% possuíam formação anterior completa ou incompleta, sendo 73,7\% estudantes de Instituição privada, conforme Tabela 1. 
Tabela 1 - Características dos sujeitos entrevistados.

\begin{tabular}{|c|c|c|c|c|c|c|c|c|}
\hline Sujeitos & Idade & Sexo & $\begin{array}{l}\text { Estado } \\
\text { Civil }\end{array}$ & IES & Período & $\begin{array}{l}\text { Cenário de } \\
\text { Prática }\end{array}$ & Disciplina & $\begin{array}{l}\text { Formação } \\
\text { Anterior }\end{array}$ \\
\hline MA & 25 & $M$ & Solteiro & Pública & $9^{\circ} \mathrm{P}$ & UBS & MIC & Não \\
\hline MB & 26 & $\mathrm{~F}$ & Solteiro & Pública & $11^{\circ} \mathrm{P}$ & UBS & MIC & Não \\
\hline MC & 25 & $\mathrm{M}$ & Solteiro & Pública & $9^{\circ} \mathrm{P}$ & UBS & MIC & Não \\
\hline MD & 23 & $\mathrm{~F}$ & Solteiro & Pública & $9^{\circ} \mathrm{P}$ & UBS & MIC & Não \\
\hline ME & 27 & $\mathrm{~F}$ & Solteira & Privada & $10^{\circ} \mathrm{P}$ & UBS & ISEC & Não \\
\hline MF & 25 & M & Solteiro & Privada & $10^{\circ} \mathrm{P}$ & UBS & ISEC & Não \\
\hline MG & 24 & $\mathrm{~F}$ & Solteira & Privada & $10^{\circ} \mathrm{P}$ & UBS & ISEC & Não \\
\hline MH & 25 & $\mathrm{~F}$ & Solteira & Privada & $10^{\circ} \mathrm{P}$ & UBS & ISEC & Sim \\
\hline MI & 27 & $\mathrm{~F}$ & Solteira & Privada & $9^{\circ} \mathrm{P}$ & UBS & ISEC & Não \\
\hline MJ & 25 & $\mathrm{~F}$ & Solteira & Privada & $10^{\circ} \mathrm{P}$ & UBS & ISEC & Não \\
\hline MK & 26 & $\mathrm{~F}$ & Solteira & Privada & $7^{\circ} \mathrm{P}$ & UBS & ISEC & Sim * \\
\hline ML & 25 & $\mathrm{~F}$ & Solteira & Privada & $6^{\circ} \mathrm{P}$ & UBS & ISEC & Não \\
\hline MM & 25 & $\mathrm{M}$ & Solteiro & Privada & $6^{\circ} \mathrm{P}$ & UBS & ISEC & Não \\
\hline MN & 26 & $\mathrm{~F}$ & Solteira & Privada & $6^{\circ} \mathrm{P}$ & UBS & ISEC & Sim \\
\hline MO & 24 & $\mathrm{~F}$ & Solteiro & Pública & $9^{\circ} \mathrm{P}$ & UBS & MIC & Não \\
\hline MP & 23 & $\mathrm{~F}$ & Solteiro & Pública & $9^{\circ} \mathrm{P}$ & UBS & MIC & Não \\
\hline MQ & 36 & $\mathrm{M}$ & Casado & Pública & $10^{\circ} \mathrm{P}$ & UBS & MIC & Sim \\
\hline MR & 25 & $\mathrm{~F}$ & Solteira & Pública & $9^{\circ} \mathrm{P}$ & $\begin{array}{l}\text { UBS } \\
\text { CAPS }\end{array}$ & MIC & Não \\
\hline MS & 23 & M & Solteiro & Pública & $10^{\circ} \mathrm{P}$ & $\begin{array}{l}\text { UBS } \\
\text { CAPS }\end{array}$ & MIC & Não \\
\hline MT & 25 & M & Solteiro & Pública & $10^{\circ} \mathrm{P}$ & $\begin{array}{l}\text { UBS } \\
\text { CAPS }\end{array}$ & MIC & Não \\
\hline MU & 24 & $\mathrm{~F}$ & Casada & Privada & $2^{\circ} \mathrm{P}$ & UBS & IESC & Não \\
\hline MV & 22 & $\mathrm{~F}$ & Solteira & Privada & $2^{\circ} \mathrm{P}$ & UBS & IESC & Não \\
\hline MW & 26 & $\mathrm{~F}$ & Solteira & Privada & $5^{\circ} \mathrm{P}$ & UBS & IESC & Sim* \\
\hline MX & 21 & $\mathrm{~F}$ & Solteira & Privada & $3^{\circ} \mathrm{P}$ & UBS & IESC & Não \\
\hline MY & 20 & $\mathrm{M}$ & Solteiro & Privada & $4^{\circ} \mathrm{P}$ & UBS & IESC & Não \\
\hline MZ & 23 & $\mathrm{~F}$ & Solteira & Privada & $5^{\circ} \mathrm{P}$ & UBS & IESC & Não \\
\hline NA & 22 & $\mathrm{M}$ & Solteiro & Privada & $5^{\circ} \mathrm{P}$ & UBS & IESC & Não \\
\hline NB & 19 & $\mathrm{~F}$ & Solteira & Privada & $3^{\circ} \mathrm{P}$ & UBS & IESC & Não \\
\hline NC & 24 & $\mathrm{~F}$ & Solteira & Privada & $3^{\circ} \mathrm{P}$ & UBS & IESC & Sim \\
\hline ND & 26 & $\mathrm{M}$ & Solteiro & Privada & $3^{\circ} \mathrm{P}$ & UBS & IESC & Não \\
\hline NE & 30 & $\mathrm{M}$ & Solteiro & Privada & $8^{\circ} \mathrm{P}$ & UBS & IESC & Sim \\
\hline NF & 24 & $\mathrm{M}$ & Solteiro & Privada & $7^{\circ} \mathrm{P}$ & UBS & IESC & Não \\
\hline NG & 22 & $\mathrm{~F}$ & Solteira & Privada & $4^{\circ} \mathrm{P}$ & UBS & IESC & Sim \\
\hline NH & 22 & $\mathrm{~F}$ & Solteira & Privada & $4^{\circ} \mathrm{P}$ & UBS & IESC & Não \\
\hline NI & 27 & $\mathrm{M}$ & Solteiro & Privada & $6^{\circ} \mathrm{P}$ & UBS & IESC & Sim \\
\hline NJ & 23 & M & Solteiro & Privada & $6^{\circ} \mathrm{P}$ & UBS & IESC & Não \\
\hline NK & 23 & $\mathrm{M}$ & Solteiro & Privada & $7^{\circ} \mathrm{P}$ & UBS & IESC & Não \\
\hline NL & 22 & $\mathrm{~F}$ & Solteira & Privada & $5^{\circ} \mathrm{P}$ & UBS & IESC & Não \\
\hline
\end{tabular}

Abreviações: UBS - Unidade Básica de Saúde; IESC - Integração ensino, serviço e comunidade, ISEC - Integração serviço, ensino e comunidade; MIC - Médico, indivíduo e comunidade; CAPS - Centro de Atenção Psicosocial.

Fonte: Autores (2021).

A partir da análise do conteúdo foi possível identificar as categorias de análise e os eixos temáticos como apresentados na Tabela 2. 
Tabela 2 - Eixos e respectivas categorias evidenciados nas gravações.

EIXO TEMÁTICO CATEGORIA DE ANÁLISE

Ações Educativas Desenvolvidas

Vivência Prática

Recursos Educativos

Integração, Ensino, Serviço e Comunidade

Trabalho em Equipe

Prevenção a Saúde

Cuidado em Saúde

Autocuidado

Benefícios das ações Educativas

Dificuldades das ações Educativas

Ações Educativas

Sugestões das ações Educativas

Fonte: Autores (2021).

O eixo temático "Vivência Prática" se refere a inserção do estudante na comunidade, durante a graduação, experenciando relações mais próximas com os pacientes, estabelecimento de vínculos, cuidado integral à saúde da população e a oportunidade de conhecer a realidade dos pacientes, familiarizando-se com o cenário. O eixo temático "Cuidado em Saúde", por sua vez, enfatiza as mudanças nos hábitos de vida e propagação de conhecimento, levando ao autoconhecimento. Por fim, o eixo temático, “Ações Educativas", aborda a importância da comunicação entre a equipe de saúde, a comunidade e os estudantes inseridos nos campos de prática.

\section{Vivência Prática: Ações Educativas Desenvolvidas, Recursos Educativos, Integração Ensino Serviço e Comunidade e Trabalho em Equipe.}

A educação em saúde é um campo de práticas e de conhecimentos do âmbito da saúde que tem se ocupado diretamente com a criação de vínculos entre a ação assistencial e o pensar e fazer cotidiano da população. As práticas de educação em saúde estimulam a reflexão da população na busca de cuidado integral, tornando o usuário atuante em seu autocuidado com autonomia (Brasil, 2015; Dias et al., 2013). Para Freire (2011) a construção da prática educativa requer troca de saberes e valorização do diálogo com compartilhamento de conhecimentos entre educador e o educando. Apesar da literatura defender um modelo dialógico, horizontal e crítico foi possível perceber a adoção de abordagens tradicionais que valorizam a transmissão da informação, como pode ser observado nas falas a seguir:

No meu cenário de prática realizamos palestras, entrega de panfletos informativos. Realizadas com supervisão de diretores e coordenadores, essas ações são voltadas para os pacientes e profissionais, visando o aprimoramento de novos conhecimentos e práticas em saúde, acho que seja isso mesmo! (MQ1, masculino, $10^{\circ}$ período).

As práticas educativas em nosso cenário de prática são: Rodas educativas, dias padronizados para discussão de temas que vemos no dia a dia o que mais acomete aqueles usuários, em salas de espera isso dependera do tema e como achamos melhor adesão do tema para aquela população (MI1, feminino, $9^{\circ}$ período).

O estudo de Brasil et al. (2018) realizado com a equipe de saúde bucal de uma unidade de saúde da família, apresentou palestras expositivas (na comunidade ou em sala de espera) com exibição de cartazes e modelos didáticos (peças 
anatômicas, instrumentos de higiene pessoal, dentre outros.), como as abordagens educacionais mais utilizadas. Corroborando com o estudo de Cabral et al (2021) que apresenta a vivência em um projeto de extensão com estudantes de medicina que realizam atividades educativas em sala de espera como oficinas, palestras, folhetos ilustrativos e orientações verbais. Não obstante haja outros processos de educação em saúde em curso, as ações educativas desenvolvidas via transmissão de informações foram prevalentes nas falas dos entrevistados que, revelaram o caráter prescritivo das escolhas de temas desenvolvidos na comunidade no ambiente da sala de espera:

A gente faz muito sala de espera, já fomos em escola também, fizemos pesquisa em uma escola e a gente tá para levar um feedback ainda dessa pesquisa, foi sobre qualidade alimentar dos alunos (NG1, feminino, $4^{\circ}$ período).

A gente teve muito contato com a sala de espera em si, a gente escolhia um tema dependendo da demanda da Unidade de Saúde. Por exemplo, se tivessem muitas mulheres grávidas naquele dia que a gente estivesse a gente falava de algum tema voltado para elas. (MZ1, feminino, $5^{\circ}$ período).

A Estratégia Saúde da Família, como o primeiro contato para os usuários, apresenta-se como o cenário privilegiado para a prática educativa por se basear em tecnologias cognitivas voltadas para a promoção da saúde e prevenção de doenças (Mendes et al., 2020; Vasconcelos et al., 2009). Logo, é importante que as ações educativas sejam ampliadas ao ambiente comunitário aproximando-se da realidade dos usuários e colaborando com a construção dialógica do saber.

O estudo de Couto et al. (2018) constatou que, ao contrário do que é proposto pelo SUS, no qual a APS deve ser a porta de entrada do usuário ao serviço de saúde, muitos usuários buscam inicialmente os níveis de atenção secundária ou terciária. Portanto, o processo educativo apresenta-se como uma estratégia de aproximação serviço, profissional e comunidade, o que permite a maior compreensão acerca do funcionamento dos serviços ofertados na RAS, como destacado na fala a seguir: "Ensinar as pessoas sobre o sistema de saúde, como ele funciona, para que serve um hospital, para o que serve uma UPA, para que serve o PSF, o que é um PSF" (MT10, masculino, $10^{\circ}$ período).

A aproximação do ensino, serviço e comunidade é evidenciada nas entrevistas como fundamental no processo de aprendizagem. Para Bomfim (2017) experiências médicas positivas, a partir da integração ensino e serviço, foram com frequência, descritas como marcantes e de grande importância para a formação pessoal e acadêmica dos participantes, firmando como experiência enriquecedora que possibilita o aprendizado por meio da teoria aliada à prática, procurando eng ajar a comunidade na intervenção do processo saúde-doença. Corroborando com Adler e Gallian (2014) que salientam a importância desta integração na formação do estudante, quando orientada e acompanhada por professores que fazem a ligação entre a teoria e a prática, pois contribui para a melhoria da saúde dos usuários.

E a gente conseguir elaborar alguma estratégia que tire essas barreias, entendeu? Que a gente possa conversar melhor com a comunidade, que eles consigam trazer mais a comunidade para perto da gente. Porque muitas vezes o próprio usuário não se sente confortável sendo atendido por estudante de medicina, acho que falta a gente conversar um pouco com eles e explicar que é necessário porque futuramente a gente que vai atender as pessoas (MX14, feminino, $3^{\circ}$ período).

Éramos divididos em grupos pra fazer atendimentos naquele local, colher as informações nós mesmos e ter uma interação maior, mais contato mesmo, com a população e com o próprio sistema de saúde, como ocorre em um local desse com prática de saúde da família em que nós podemos ter um contato verdadeiro como vai ser toda essa questão (MM11, masculino, $6^{\circ}$ período).

É importante reforçar que a aproximação precoce entre os estudantes de Medicina e a realidade do SUS favorece a troca de experiência e saberes, sendo fundamental para compreensão da organização do sistema de saúde e para reflexão sobre o cuidado médico (Brandão et al., 2013). Ademais, essa inserção precoce também possibilita a aproximação e o 
desenvolvimento de ações educativas da equipe multiprofissional pautada na relação ética e compromissada com a comunidade, valorizando o fazer de cada profissão, contribuindo para o cuidado integral de qualidade (Cavalcante et al., 2018). O que é evidenciada em muitas falas, a importância do trabalho em equipe:

Primeiro, porque a gente tem mais contato com a população que é assistida, a gente consegue desenvolver uma união maior entre os membros da equipe seja o enfermeiro, o dentista, os agentes comunitários, a recepcionista enfim todo mundo acaba meio que participando porque a gente sempre precisa da ajuda de mais alguém (MF10, masculino, $10^{\circ}$ período).

O estudo de Barreto et al. (2019), aponta que os profissionais entrevistados destacam que: "cada indivíduo tem contribuições para esses momentos de formação, pois cada sujeito traz suas experiências e conhecimentos para as práticas, dando mais qualidade aos atendimentos educativos". Assim, a mudança no modo de agir e compreender o processo de trabalho educativo precisa ser temática de reflexão, também, entre os trabalhadores que compõem a equipe, para que o cuidado seja peculiar ao saber-fazer dos profissionais e obstáculos, como práticas verticalizadas, posturas autoritárias e ações não desenvolvidas sejam superados (Ayres, 2009).

\section{Cuidados em Saúde: Prevenção à saúde e Autocuidado}

A prevenção à saúde pode ser dividida em quatro níveis: o primário, que busca a promoção e a proteção de saúde, a exemplo da vacinação; o secundário, que visa o rastreio e diagnóstico precoce; o terciário, que tenta reduzir danos e reabilita; o quaternário, que preza pela redução de procedimentos e intervenções desnecessários (Brasil, 2017). As falas dos discentes salientaram as ações preventivas, em seus diversos níveis, bem como a relação do autocuidado com tais práticas:

E até mesmo educar quanto a prevenção, principalmente prevenção primária e secundária, que é onde eles devem estar mais atentos, digamos assim, que se a gente tivesse uma prevenção primária e secundária eficientes nós teríamos muito menos agravos (MT11, masculino, $10^{\circ}$ período).

É um Processo educativo de construção de conhecimentos em saúde, práticas para contribuir que o indivíduo consiga ter autonomia no próprio cuidado individual e coletiva (MI4, feminino, $9^{\circ}$ período).

Moraes \& Rodriges (2021) apresentam a roda de conversa com uma potente ferramenta para favorecer o diálogo, incentivar o protagonismo e conhecer o "saber em saúde" dos usuários. Logo, destaca-se a importância de práticas educativas dialogadas que estimulem o desenvolvimento da autonomia do usuário. Desta maneira, é preciso que a formação de médicos priorize o modelo de atenção integral à saúde, ou seja, foque na promoção de saúde e prevenção de agravos, além de estimular a reflexão e a consciência crítica das pessoas sobre seus problemas em saúde (Falkenberg et al., 2014).

\section{Ações Educativas: Benefícios, Dificuldades e Sugestões de Melhoria.}

Proporcionar a inserção precoce de estudantes de medicina na APS, contribui com o enfretamento de barreiras históricas na formação em saúde, relacionadas ao atendimento individual e curativo. Assim, a vivência precoce, estimula o olhar crítico e reflexivo frente às situações-problemas (Brandão et al., 2013). Ademais, foi possível perceber nas falas dos estudantes os benefícios da inserção destes no cenário da APS, conforme as unidades de registro a seguir:

Acho que, tipo, dá uma visão de mundo maior e torna a gente médicos mais humanizados também (NB4, feminino, $3^{\circ}$ período). 
Um vínculo maior com o paciente, você o acompanha, tem uma abertura maior para explicar, e facilita a adesão ao tratamento que é o principal e a relação médico-paciente acaba se fortalecendo, também (MP7, feminino, $9^{\circ}$ período).

Gonçalves et al. (2018), reforçam que o modelo de atenção ao longo do curso permite que o estudante pratique o cuidado horizontal, distanciando-se do modelo focado na doença e em suas causas biológicas, facilitando a criação do vínculo e a comunicação efetiva com os pacientes. Entretanto, é importante destacar que, quando perguntados sobre as dificuldades encontradas na realização das ações educativas, muitos discentes citaram questões estruturais, resistência dos profissionais de saúde em receber os estudantes e falta de adesão do público-alvo.

Muitas vezes as pessoas que queremos que escutem não estão presentes na atividade. Ou por falta de interesse ou porque não foram informados sobre a atividade. Por exemplo, uma vez eu fui fazer uma atividade para gestantes e só tinha uma lá, a atividade foi boa, mas poderia ter alcançado mais pessoas. Aí é um problema (MW7, feminino, $5^{\circ}$ período).

Acho que dependendo da unidade de saúde tem alguns próprios profissionais que não gostam da presença do estudante, não sei se por qualquer outro motivo, mas eles não se sentem confortáveis junto de estudantes no processo de prática, né. Tanto no consultório ou mesmo que a gente faça alguma ação de sala de espera. Então acho que esse é o maior desafio (MX10, feminino, $3^{\circ}$ período).

Bom...às vezes, são dificuldades estruturais da própria unidade também (NA8, masculino, $5^{\circ}$ período)

Tais achados concordam com o estudo Mendes et al. (2020) que apresenta desafios que devem ser superados quanto a integração ensino e serviço de saúde, dentre os quais: os inerentes ao processo de formação, aos serviços de saúde e à estrutura da universidade, bem como os desafios relacionados aos atores envolvidos no processo de formação, quer sejam docentes, discentes, preceptores e/ou a comunidade. Após a identificação das principais dificuldades, os estudantes foram estimulados a apresentar sugestões para o enfretamento dos problemas elencados, assim foi possível destacar as seguintes falas:

Que os Profissionais sejam mais acessíveis e que possamos criar um vínculo maior com a população para que ela se sinta bem em ir até aquela unidade de saúde, melhorando o empenho de toda equipe que faz parte da UBS para que essa boa relação sobressaia e seja transmitida para os usuários facilitando todo processo de adesão daquelas dicas, teorias faladas nas ações educativas (MI8, feminino, $9^{\circ}$ período).

Minha sugestão seria mesmo, essa... tem a parte financeira que é importante, ter algum incentivo financeiro que, às vezes, é bem dificultado, mas eu acho que não é o principal porquê de uma forma ou de outra eu acho que o SUS recebe dinheiro que dá pra fazer muitas coisas, só que esse dinheiro acaba sendo não usado ou usado de outra forma. E aí vai pra questão de gestão como eu tava dizendo, então a sugestão seria mesmo que o líder principal daquele postinho fosse uma pessoa muito ativa, empenhada, comprometida em fazer aquilo dá certo (MJ16, feminino, $10^{\circ}$ período).

O modelo tradicional das práticas de Medicina tem mostrado falhas quanto à comunicação com o paciente, assim é preciso investir desde a graduação no compartilhamento de saberes e no entendimento entre os envolvidos na interlocução (Araújo et al., 2015; Coriolano-Marinus et al., 2014). Desta forma, a integração da universidade com os serviços de saúde, impulsiona a formação crítica e amplia a visão do estudante, valorizando o cuidado integral e não apenas na doença e na cura.

As ações educativas são capazes de gerar benefícios a todos envolvidos, pois por meio do diálogo a equipe pode possibilitar maior vínculo com a população e maior adesão do usuário aos serviços de saúde (Santos et al., 2020; Torres et al., 2018). Quanto à competência educação em saúde os estudantes se avaliaram muito bem no aprendizado com o trabalho em equipe, reforçando o modelo de atenção à saúde da família na APS. Gonçalves et al. (2018) evidenciou que os estudantes têm a possibilidade de usufruir de um campo de prática, além de adquirir uma visão médica voltada à promoção de saúde e não 
apenas aos processos curativos. Enquanto os pacientes podem usufruir de um serviço e de uma equipe de saúde que entende suas necessidades, adequa seus atendimentos à realidade da comunidade envolvida, respeitando suas individualidades.

\section{Considerações Finais}

Neste estudo foi possível evidenciar que a educação em saúde proporcionou aproximação da realidade da comunidade, criação do vínculo com os usuários e equipe de saúde, valorização do trabalho em equipe e reconhecimento do papel do médico neste cenário. Em contrapartida, limitações e desafios se revelaram nas falas dos estudantes tais como: resistência dos profissionais de saúde em receber os estudantes, precariedade da estrutura física e baixa adesão dos usuários as práticas educativas. Portanto, espera-se que este estudo contribua com a discussão acerca dos desafios enfrentados pela formação médica para as práticas educativas na APS, pois apresenta as potencialidades e fragilidades destas práticas.

Diante das evidências debatidas e das fragilidades levantadas recomendamos para investigações futuras uma análise da percepção do profissional do serviço e do docente frente a práticas educativas e a relação ensino serviço, visto que neste estudo não foi possível realizar tal análise. Este estudo delimitou-se a estudantes de universidades públicas e privadas de um munícipio do Nordeste, assim sugerimos que futuros estudos apresentem uma maior diversidade de cenários acadêmicos no que se refere a formação médica para as práticas educativas na APS.

\section{Referências}

Adler, M. S., \& Gallian, D. M. C. (2014). Formação médica e serviço único de saúde: propostas e práticas descritas na literatura especializada. Revista Brasileira de Educação Médica, 38(3), 388-396. https://doi.org/10.1590/s0100-55022014000300014

Araújo, E. P. S., Cruz, P. J. S. C, Alencar, I. C., Carneiro, D. G. B. (2015). Educação Popular no Processo de Integração Ensino Serviço e Comunidade: Reflexões com Base em Experiências na Extensão. Revista de Aps. 18(4), 447-455.

Ayres, S. R. C. M. (2009). Cuidado: trabalho e interação nas práticas de saúde. Rio de Janeiro: Abrasco.

Bardin, L. (1977). Análise de conteúdo. Lisboa: Edições 70.

Barreto, A. C. O., Rebouças, C. B. de A., Aguiar, M. I. F. de, Barbosa, R. B., Rocha, S. R., Cordeiro, L. M., Melo, K. M. de, \& Freitas, R. W. J. F. de. (2019). Perception of the Primary Care multiprofessional team on health education. Revista Brasileira de Enfermagem, 72, 266-273. https://doi.org/10.1590/00347167-2017-0702

Bomfim, A. M. A. (2017). Relações interpessoais no desenvolvimento da integração ensino -serviços de saúde. Universidade Federal de São Paulo.

Bosi, M.L.M, Mercado, F.J. (2004). Pesquisa qualitativa de serviços de saúde. Petrópolis: Vozes.

Brandão, E. R. M., Rocha, S. V., \& Silva, S. S. da. (2013). Práticas de integração ensino-serviço-comunidade: Reorientando a formação médica. Revista Brasileira de Educação Médica, 37(4), 573-577. https://doi.org/10.1590/s0100-55022013000400013

Brasil, P. R. D. C., \& Santos, A. M. DOS. (2018). Desafios às ações educativas das Equipes de Saúde Bucal na Atenção Primária à Saúde: táticas, saberes e técnicas. Physis: Revista de Saúde Coletiva, 28(4), 1-23. https://doi.org/10.1590/s0103-73312018280414

Brasil. (2015). Portaria n ${ }^{\circ} 1130$, de 05 de agosto de 2015. Institui a Política Nacional de Atenção Integral à Saúde da Criança (PNAISC) no âmbito do Sistema Único de Saúde (SUS). Diário Oficial da União. Brasília, DF, 06 ago. 2015. 149 (Seção 1), 37-39.

Brasil. (2017). Portaria no 2.436, de 21 de setembro de 2017. Aprova a Política Nacional de Atenção Básica, estabelecendo a revisão de diretrizes para a organização da Atenção Básica, no âmbito do Sistema Único de Saúde (SUS). Diário Oficial da União. 183. ed. Brasília, DF, 22 set. 2017. Seção 1, 68-76.

Cabral, D. A. C., Silva, M. C. P. da, Campos, F. M. S., Medeiros, J. P. do V., Batista, P. dos S., Cabral, L. G. C., Hamoy, M., \& Mello, V. J. de. (2021). Educação em saúde para pacientes diabéticos em vulnerabilidade socioeconômica no norte do Brasil. Research, Society and Development, 10(1), e10910111598. https://doi.org/10.33448/rsd-v10i1.11598

Cavalcante, T. M., Melo, B. T. de, Batista, R. S. de L., Jordão, D. A., Beserra, K. S., Andrade, L. S. G. de, Lima Junior, R. C. C. de, \& Bomfim, A. M. A. (2018). Uma Experiência de Integração Ensino, Serviço e Comunidade de Alunos do Curso de Graduação em Medicina na Atenção Básica no Município de Maceió. Revista Ciência Plural, 3(3), 69-80.

Coriolano-Marinus, M. W. de L., de Queiroga, B. A. M., Ruiz-Moreno, L., \& de Lima, L. S. (2014). Comunicação nas práticas em saúde: Revisão integrativa da literatura. Saude e Sociedade, 23(4), 1356-1369. https://doi.org/10.1590/S0104-12902014000400019

Couto, V. B. M., Santos, C. M. B., Sampaio, B. P., Almeida, I. S. de, Medeiros, S. C., Santos, N. G. S., Menezes, T. A. M., Santos, D. C., Coelho, F. L. P., Correia, G. S., \& Guzman, J. L. D. (2018). Vivenciando a Rede: Caminhos para a Formação do Médico no Contexto do SUS. Revista Brasileira de Educação Médica, 42(2), 5-14. https://doi.org/10.1590/1981-52712015v42n2rb2016107 
Research, Society and Development, v. 10, n. 7, e9010716369, 2021

(CC BY 4.0) | ISSN 2525-3409 | DOI: http://dx.doi.org/10.33448/rsd-v10i7.16369

Dias, G. A. R., \& Lopes, M. M. B. (2013). Educação e saúde no cotidiano de enfermeiras da atenção primária. Revista de Enfermagem Da UFSM, 3(3). https://doi.org/10.5902/217976927846

Falkenberg, M. B., Mendes, T. de P. L., de Moraes, E. P., \& de Souza, E. M. (2014). Educação em saúde e educação na saúde: Conceitos e implicações para a saúde coletiva. Ciencia e Saude Coletiva, 19(3), 847-852. https://doi.org/10.1590/1413-81232014193.01572013

Feuerwerker, L. C. M. (2014). Micropolítica e a formação de profissionais de saúde. In Micropolítica e saúde: produção do cuidado, gestão e formação (1st ed.). Editora Rede Unida. www.redeunida.org.br

Freire, P. (2011). Pedagogia do oprimido. (50 ed.). Rio de Janeiro: Paz e Terra.

Gonçalves, J. V., Silva, R. F. da, \& Gonçalves, R. de C. (2018). Cuidado à Saúde e a Formação do Profissional Médico. Revista Brasileira de Educação Médica, 42(3), 9-15. https://doi.org/10.1590/1981-52712015v42n3rb20180029r1

Mendes, T. de M. C., Ferreira, T. L. dos S., Carvalho, Y. de M., Silva, L. G. da, Souza, C. M. C. de L., \& Andrade, F. B. de. (2020). Contributions and Challenges of Teaching-Service-Community Integration. Texto \& Contexto - Enfermagem, 29. https://doi.org/10.1590/1980-265x-tce-2018-0333

Minayo, M. C. S. (2011). Pesquisa Social: teoria, método e criatividade (30 ed.). Petrópolis: Vozes.

Miranda, G. M. D., Mendes, A. da C. G., \& Silva, A. L. A. da. (2016). Desafios das políticas públicas no cenário de transição demográfica e mudanças sociais no Brasil. Interface - Comunicação, Saúde, Educação, 21(61). https://doi.org/10.1590/1807-57622016.0136

Moraes, M. C. L. de, \& Rodrigues, D. K. de B. (2021). Roda de conversa no CECCO: uma experiência. Research, Society and Development, 10(3), e36810313510. https://doi.org/10.33448/rsd-v10i3.13510

Nutbeam, D. (2018). Health education and health promotion revisited. Health Education Journal, 78(6), 705-709. https://doi.org/10.1177/0017896918770215

Ribeiro, K. S. Q. S. (2013). Fisioterapia e educação popular em saúde: mudança epistemológica e reorientação da prática. In J. P. Bispo Júnior (Ed.), Fisioterapia e Saúde Coletiva: Reflexões, fundamentos e desafios. (1st ed., pp. 98-127). São Paulo: Hucitec Editora.

Santos, F. F. dos, Miranda, C. Z. de, Pertile, K. C., Barbosa, M. S., Caldeira, A. P., \& Costa, S. de M. (2020). Desempenhos na Área de Competência Educação em Saúde: Autoavaliação de Estudantes de Medicina. Revista Brasileira de Educação Médica, 44(3). https://doi.org/10.1590/1981-5271v44.320190291

Soares, A. N., Souza, V. de, Santos, F. B. O., Carneiro, A. C. L. L., \& Gazzinelli, M. F. (2017). Dispositivo Educação em Saúde: Reflexões sobre Práticas Educativas na Atenção Primária e Formação em Enfermagem. Texto \& Contexto - Enfermagem, 26(3). https://doi.org/10.1590/0104-07072017000260016

Torres, H. de C., Pace, A. E., Chaves, F. F., Velasquez-Melendez, G., \& Reis, I. A. (2018). Evaluation of the effects of a diabetes educational program: A randomized clinical trial. Revista de Saude Publica, 52. https://doi.org/10.11606/S1518-8787.2018052007132

Vasconcelos, M., Grillo, M.J.C., Soares, S.M., (2009). Práticas Pedagógicas em Atenção Básica à Saúde. Tecnologias para abordagem ao indivíduo, família e comunidade. [NESCON/UFMG - Curso de Especialização em Atenção Básica em Saúde da Família]. Belo Horizonte: Nescon/UFMG, 73p.

Vendruscolo, C., Prado, M. L. do, \& Kleba, M. E. (2014). Formação de recursos humanos em saúde no Brasil: uma revisão integrativa. Educação Em Revista, 30(1). https://doi.org/10.1590/S0102-46982014000100009 